\title{
INTERFERÊNCIA DA LIGNINA NA QUANTIFICAÇÃO DE RADICAIS LIVRES NO PROCESSO DE COMPOSTAGEM
}

\author{
Lucimar Lopes Fialho* \\ Embrapa Instrumentação Agropecuária, CP 741, 13560-970 São Carlos - SP / Instituto de Química de São Carlos, Universidade \\ de São Paulo, CP 780, 13560-250 São Carlos - SP, Brasil \\ Wilson Tadeu Lopes da Silva, Débora M. B. P. Milori, Marcelo Luiz Simões e Ladislau Martin-Neto \\ Embrapa Instrumentação Agropecuária, CP741, 13560-970 São Carlos - SP, Brasil \\ Sérgio da Costa Saab \\ Departamento de Física, Universidade Estadual de Ponta Grossa, CP 4748, 84030-000 Ponta Grossa - PR, Brasil
}

Recebido em 24/3/09; aceito em 5/8/09; publicado na web em 11/1/10

\begin{abstract}
INTERFERENCE OF LIGNIN IN THE QUANTIFICATION OF FREE RADICALS IN THE PROCESS OF COMPOSTING. In this work was studied composts and their humic acids (HA) to evaluate the use of EPR in the monitoring of the humification. It was observed increase in the concentration of organic free radical (OFR) in all the composts, but in L1 the increase was more significant than L2 and L3. Research more detailed of L1 showed that the lignin is main source of OFR. Then determination of these radicals in material in nature that contains high amount of lignin isn't a good indicator to monitor the humification process in the composting and yes the fraction HA of the composts.
\end{abstract}

Keywords: composting; lignin; EPR.

\section{INTRODUÇÃO}

O processo de compostagem pode ser considerado uma versão acelerada do processo natural de transformação da matéria orgânica no solo, sendo obtido por meio do fornecimento de condições favoráveis (como temperatura, umidade, $\mathrm{pH}$ e aeração) à atividade microbiana. ${ }^{1}$ A decomposição biológica depende ainda da razão de degradação dos compostos de carbono presentes na amostra (carboidratos, aminoácidos, ácidos graxos, celulose, lignina etc), bem como seu conteúdo de nutrientes. ${ }^{2}$

Os materiais lignocelulósicos, como a madeira, são constituídos principalmente pela mistura de celulose $(\sim 40 \%)$, hemicelulose (de 20 a $30 \%$ ) e lignina (de 20 a 30\%). Sendo a lignina um dos constituintes da planta de mais difícil degradação. ${ }^{3}$

A lignina é um polímero derivado de grupos fenilpropanoides e é formada a partir de três precursores básicos: os álcoois $p$-cumarílico, coniferílico e sinapílico (Figura 1).
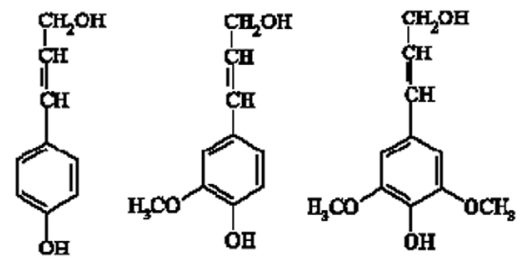

Figura 1. Precursores básicos na formação da molécula de lignina. Adaptada da ref. 4

López et al..$^{5}$ consideram que as mudanças sofridas pelos materiais contendo lignina e celulose durante as transformações que ocorrem no solo, ou no processo de compostagem, são as principais responsáveis pela formação das substâncias húmicas (SH). Isso porque para a molécula de lignina ser incorporada à estrutura das SH ela deve passar por reações químicas de degradação e, quanto maior for essa degradação,

*e-mail: fialholl@yahoo.com.br maior será sua contribuição na formação da estrutura das SH.

A espectroscopia de ressonância paramagnética eletrônica (RPE) é uma técnica usada nas análises das SH para identificar radicais livres do tipo semiquinona (RLO) e metais de transição. ${ }^{6-8}$ A concentração dos RLO tem sido usada como um índice de humificação da matéria orgânica de solos, sendo que o aumento da concentração desses radicais está diretamente associado ao grau de humificação. ${ }^{9-12}$ Seu valor pode mudar quando se varia o $\mathrm{pH}$ da amostra em solução, quando a SH é submetida ao processo de hidrólise ácida, quando se altera a temperatura, quando há processo redox, quando a SH é irradiada por luz UV e na presença de íons paramagnéticos. ${ }^{13-15}$

Nesse trabalho, buscou-se aplicar essa técnica na avaliação do grau de humificação da matéria orgânica de compostos, observando a potencialidade do seu uso para a quantificação de RLO em determinadas misturas de resíduos orgânicos e identificar possíveis interferentes nessa quantificação, pois sabe-se que a lignina contém RLO em concentrações comparáveis com a observada em materiais húmicos. ${ }^{16}$ Portanto, sua presença pode influenciar na quantificação de RLO e, consequentemente, na determinação do processo de humificação da matéria orgânica dos compostos, produzidos a partir de resíduos com alto teor de lignina. Dessa forma, esse trabalho teve como objetivo avaliar a influência da lignina na quantificação de RLO durante o monitoramento do processo de compostagem, a partir de diferentes resíduos orgânicos.

\section{PARTE EXPERIMENTAL}

\section{Montagem do experimento}

O experimento foi montado em um pátio de superfície de concreto na Fazenda Santa Cândida, município de São Carlos - SP, no dia 25/10/2004 e monitorado durante 7 meses.

Inicialmente os resíduos foram caracterizados quanto à umidade e ao teor de C e N. Em função desses dados foram feitos os cálculos das proporções dos resíduos a fim de que todos os tratamentos (exceto L1) tivessem uma relação $\mathrm{C} / \mathrm{N}$ inicial de 35 a 40. 
As proporções dos resíduos em cada leira, considerando a massa fresca, foram de, aproximadamente: Leira $1(\mathrm{~L} 1)=900 \mathrm{~kg}$ de poda de árvores; Leira $2(\mathrm{~L} 2)=1450 \mathrm{~kg}$ de poda de árvores $+2450 \mathrm{~kg}$ de esterco bovino fresco; Leira $3(\mathrm{~L} 3)=1400 \mathrm{~kg}$ de poda de árvores + $2900 \mathrm{~kg}$ de bagaço de laranja triturado.

A poda de árvores, o esterco bovino fresco e o bagaço de laranja apresentavam teor de umidade de 63, 80 e $80 \%$, respectivamente. Logo, a proporção dos resíduos, considerando a massa seca, nas leiras L2 e L3 foi de, aproximadamente, 1:1. A poda de árvores foi utilizada como agente estruturante em todas as pilhas.

Um molde de madeira, com as dimensões: $2,10 \times 1,40 \times 1,22 \mathrm{~m}$, de comprimento, largura e altura, respectivamente, foi utilizado com o objetivo de padronizar o volume das leiras. Os resíduos foram misturados e colocados nesse molde até completar o volume; em seguida, o molde foi desmontado e a leira foi estruturada em formato cônico.

Foi feito monitoramento semanal do teor de umidade que era ajustado para a faixa de 50 a $60 \%$ durante todo o processo. As leiras eram cobertas com lonas plásticas nos períodos de chuva, para evitar excesso de umidade nos resíduos. Com o objetivo de fornecer $\mathrm{O}_{2}$ aos micro-organismos no interior das leiras elas foram revolvidas manualmente uma vez por semana. A partir da $14^{\mathrm{a}}$ semana as leiras não foram mais reviradas, pois a temperatura no interior das mesmas já se encontrava na fase de resfriamento e maturação.

Medidas de temperatura foram feitas diariamente, entre 6 e $7 \mathrm{~h}$ da manhã, utilizando-se um termopar do tipo K e um medidor marca Minipa, acoplado a uma haste de PVC com, aproximadamente, $50 \mathrm{~cm}$ de comprimento. As medidas foram feitas introduzindo-se essa haste no interior das leiras em três pontos distintos e a partir desses dados determinou-se a temperatura média no interior de cada leira.

Várias subamostras foram coletadas em pontos aleatórios no interior das leiras, misturadas e homogeneizadas, obtendo-se uma amostra composta de, aproximadamente, $1,0 \mathrm{~kg}$ em cada coleta. Essas coletas foram feitas nos tempos: $0,30,60,90,120,150,180$ e 210 dias de compostagem. As amostras foram secas em estufa a $60{ }^{\circ} \mathrm{C}$ até obter massa constante e, em seguida, foram trituradas em moinho de rotor vertical com facas (Tipo Willye), marca Solab, passando por peneira de $0,5 \mathrm{~mm}$.

\section{Análises químicas e espectroscópicas}

\section{Relação C/N dos compostos}

As análises de carbono total foram feitas em um aparelho TOCVCPH Shimadzu, acoplado a um módulo para amostras sólidas SSM5000A Shimadzu, com detector de combustão e a determinação de N total foi feita pelo método de Kjeldahl. ${ }^{17} \mathrm{~A}$ relação $\mathrm{C} / \mathrm{N}$ é calculada como sendo a razão atômica do C e N.

\section{Relação da capacidade de troca catiônica pelo teor de carbono} (CTC/C) dos compostos

A determinação da CTC foi feita baseada na metodologia de Rodella e Alcarde ${ }^{18}$ que fizeram uma adaptação da metodologia da Association of Official Analytical Chemists empregada para análise de CTC de turfa. ${ }^{19}$

\section{Extração dos ácidos húmicos $(A H)$}

A extração dos $\mathrm{AH}$ dos compostos foi realizada seguindo a metodologia sugerida pela Sociedade Internacional de Substâncias Húmicas (IHSS) para extração de $\mathrm{AH}$ de solos. ${ }^{20}$.

$\mathrm{Na}$ literatura ainda não há um consenso quanto ao nome dado ao material extraído dos compostos, alguns autores chamam de ácidos húmicos, enquanto outros dão a denominação de humic-like substances. ${ }^{2}$ Porém, nesse trabalho, optou-se por chamar de ácidos húmicos $(\mathrm{AH})$ todo o material extraído dos compostos, utilizando o método sugerido pela IHSS. Os AH foram submetidos à diálise em membranas Spectra/Por ${ }^{\circledR} 6000-8000$ Da que foram preparadas para o uso segundo metodologia descrita por McPhie. ${ }^{21} \mathrm{Em}$ seguida, os AH foram liofilizados e analisados por espectroscopia de ressonância paramagnética eletrônica (RPE) e fluorescência.

\section{Teor de $C$ dos $A H$}

A determinação do teor de $\mathrm{C}$ dos $\mathrm{AH}$ foi feita a partir de $1 \mathrm{mg}$ de amostra, por combustão a $1000{ }^{\circ} \mathrm{C}$. As medidas foram realizadas no equipamento Carlo-Erba -Instruments modelo EA 1110.

\section{Obtenção da lignina pelo método Klason}

Esse método consiste em uma hidrólise ácida do material, onde os polissacarídeos são removidos e a lignina é obtida como o resíduo.

Fez-se um tratamento prévio das amostras do composto da L1 para retirar os possíveis AH da amostra antes da obtenção da lignina, pois os RLO dos AH poderiam interferir na quantificação dos RLO da lignina. Aproximadamente $45 \mathrm{~g}$ do composto da L1 nos tempos zero e com 210 dias de compostagem foram submetidos a cinco extrações sequenciais dos $\mathrm{AH}$ com, aproximadamente, $600 \mathrm{~mL}$ de solução de $\mathrm{NaOH} 0,1 \mathrm{~mol} \mathrm{~L}^{-1}$, para cada extração. $\mathrm{O}$ procedimento foi repetido cinco vezes justamente para garantir a máxima extração dos $\mathrm{AH}$ presentes nos compostos. Após as extrações o material insolúvel foi seco e, aproximadamente, 1,5 g utilizado para obtenção da lignina. Utilizou-se o método TAPPI ${ }^{22} \mathrm{e}$ após ser seca em estufa a $100{ }^{\circ} \mathrm{C}$ por $2 \mathrm{~h}$, a lignina foi armazenada para posterior análise por RPE.

\section{Ressonância paramagnética eletrônica (RPE)}

Os espectros foram obtidos a partir das amostras do material in natura (composto apenas seco e moído), da lignina obtida do composto da L1 no tempo inicial $\left(\mathrm{t}_{0}\right)$ e no tempo final $\left(\mathrm{t}_{210}\right)$ de compostagem e dos AH extraídos dos compostos. As análises foram feitas seguindo o procedimento descrito por Martin-Neto et al...23

As medidas dos radicais livres do tipo semiquinona (RLO) foram feitas em um espectrômetro Bruker modelo EMX que opera em Banda $\mathrm{X}$ e os parâmetros empregados nas medidas foram: frequência de modulação $=100 \mathrm{KHz}, \mathrm{H}_{0}=340 \mathrm{mT}, \Delta \mathrm{H}=10 \mathrm{mT}, v=9,5 \mathrm{GHz}$, potência de micro-ondas $=0,2 \mathrm{~mW}$ no material in natura e $0,127 \mathrm{~mW}$ nos AH (valores obtidos a partir da curva de saturação de potência), amplitude de modulação $=0,1 \mathrm{mT}$ pico a pico, número de varreduras $=16$, ganho $=1 \times 10^{4}$ e temperatura ambiente

A concentração dos RLO foi determinada usando a aproximação $\mathrm{I} \times(\Delta \mathrm{H})^{2}$, onde $\Delta \mathrm{H}$ é a largura de linha tomada pico a pico e I é a amplitude do sinal de RPE, ${ }^{24}$ tendo como padrão de concentração o strong pitch, um material que possui uma concentração conhecida de

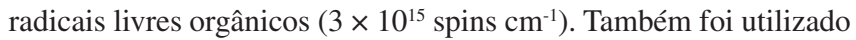
um cristal de rubi como padrão secundário para detectar possíveis alterações no fator $\mathrm{Q}$ da cavidade ressonante. As concentrações dos RLO foram corrigidas em função do teor de carbono das amostras e expressos em spins $\left(\mathrm{g} \mathrm{C}^{-1} \times 10^{17}\right.$.

\section{Análises de fluorescência}

As análises de fluorescência foram realizadas em um espectrômetro de luminescência Perkin Elmer LS-50B. Foram utilizadas soluções de $20 \mathrm{mg} \mathrm{L}^{-1}$ de $\mathrm{AH}$ (em solução aquosa de $\mathrm{NaHCO}_{3} 0,05$ mol L $\left.{ }^{-1}\right)$. A abertura das fendas de entrada e saída foi de $10 \mathrm{~nm}$ e a velocidade de varredura dos espectros foi de $200 \mathrm{~nm} \mathrm{~min}$. A varredura foi no modo de emissão, com excitação em $465 \mathrm{~nm}$. $\mathrm{O}$ cálculo do índice $\mathrm{A}_{465}$ foi realizado segundo a metodologia proposta por Milori et al... 


\section{RESULTADOS E DISCUSSÃO}

\section{Análises de RPE do composto e da lignina}

Foram determinadas as concentrações dos RLO e a largura de linha do sinal do material in natura a fim de avaliar a possibilidade do uso dessa técnica para a determinação do grau de humificação da matéria orgânica de compostos.

Na Figura 2 são apresentados os espectros dos RLO do composto da L1 e as larguras de linha $(\Delta \mathrm{H})$ dos compostos da L1, L2 e L3 em função do tempo de compostagem. Foram observadas variações nas intensidades dos sinais (Figura 2a), em função do tempo, mas não se observam grandes variações na largura de linha (Figura 2b). Apenas o composto da L3, no tempo zero de compostagem, apresentou um baixo valor na largura de linha. Isso pode ser devido a dois fatores: baixa concentração de RLO nesse material $\left(0,3 \times 10^{17}\right.$ spins $\left.(\mathrm{g} \mathrm{C})^{-1}\right)$ e também ao baixo valor de $\mathrm{pH}$ $(4,0)$ nesse tempo de compostagem, em comparação aos demais tempos e às outras leiras ( $\mathrm{pH}$ próximo de 7,0). Senesi e Schnitzer ${ }^{26}$ observaram redução na largura de linha de alguns ácidos fúlvicos quando medidos em $\mathrm{pH}$ 2,2 comparado com esses mesmos materiais em $\mathrm{pH}$ 7,0.
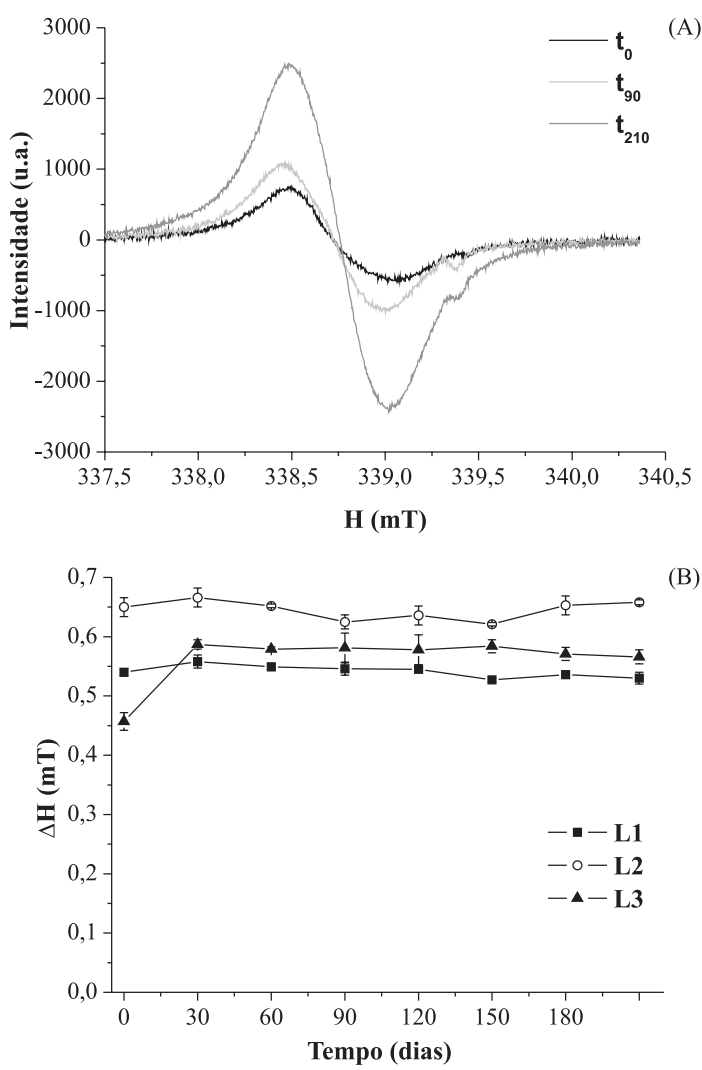

Figura 2. Sinais de radicais livres do tipo semiquinona ( $R L O)$ do composto da L1 em função do tempo de compostagem (a), largura de linha tomada pico a pico $(\Delta H)$ do sinal de RLO dos compostos da L1, L2 e L3, em função do tempo (b)

As concentrações dos RLO nos compostos, normalizadas pelo teor de carbono, em função do tempo são apresentadas na Figura 3. Observa-se incremento nas concentrações desses radicais livres em função do tempo de compostagem, sendo um dos aumentos mais pronunciado observado para o composto da L1, sugerindo que esse composto era o mais humificado. Porém, pelo monitoramento da razão atômica $\mathrm{C} / \mathrm{N}, \mathrm{CTC} / \mathrm{C}$ dos compostos, análise de fluorescência dos AH (Tabela 1), concentração dos RLO dos AH (Figura 6) e acompanhamento da temperatura no decorrer do processo observou-se que o composto da L1 não indicava características de material humificado.

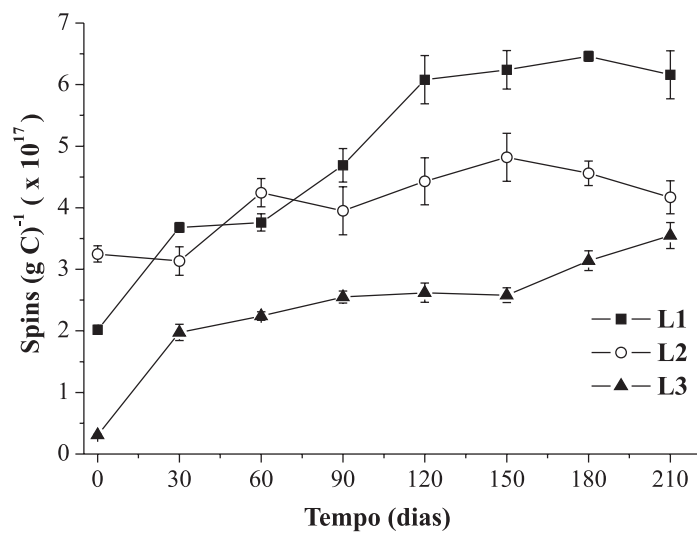

Figura 3. Concentrações de RLO em spins $(\mathrm{g} \mathrm{C})^{-1}\left(\times 10^{17}\right)$ dos materiais in natura das leiras L1, L2 e L3 em função do tempo de compostagem, determinadas por RPE

Pela análise da Tabela 1 observa-se que a relação $\mathrm{C} / \mathrm{N}$ na L1, mesmo com 210 dias de compostagem, foi de 37,9 (valor considerado na literatura muito alto para um material humificado), enquanto que na L2 e L3 foi de 15 e 17,2, respectivamente, valores considerados na faixa de material humificado. ${ }^{27} \mathrm{~A} \mathrm{CTC} / \mathrm{C}$ da L1 não atingiu o valor mínimo recomendado pela Instrução Normativa n ${ }^{\circ} 23$, de 31/8/2005, do Ministério de Agricultura, Pecuária e Abastecimento para fertilizantes orgânicos, que corresponde a $2,0 \mathrm{mmol}_{\mathrm{c}} \mathrm{g}^{-1} 28$ e o índice $\mathrm{A}_{465}$ obtido a partir dos espectros de fluorescência mostra valores com tendência à queda na L1, enquanto que na L2 e L3 há aumento desse índice. $\mathrm{O}$ aumento do índice está diretamente relacionado com o avanço da humificação da matéria orgânica, como mostrado por Milori et al., ${ }^{25}$ sugerindo assim que apenas nas leiras L2 e L3 houve realmente humificação.

Pelo monitoramento da temperatura, a L1 permaneceu durante todo o período na fase mesofílica, enquanto que na L2 e L3 a temperatura alcançou os $60^{\circ} \mathrm{C}$ (valor característico da fase termofílica, indicando ação efetiva dos micro-organismos sob os resíduos).

Dessa forma, todos os parâmetros avaliados indicam que a L1 não atingiu a humificação. Tem-se como hipótese que a maior concentração dos radicais livres no material in natura da L1 é devido aos RLO da lignina presente na poda de árvores e o aumento nessa concentração, durante o período estudado, ocorreu possivelmente em função das alterações da lignina e não pode ser relacionado ao processo de humificação, como demonstraram os outros parâmetros avaliados. Para Czechowski et al., ${ }^{16}$ a concentração de RLO na lignina é alta e comparável à observada em materiais humificados. Nas leiras L2 e L3 havia menos lignina no início do processo, comparando com a L1, pois houve mistura de outros resíduos (na proporção de, aproximadamente, 1:1 em massa seca, como foi descrito na montagem do experimento).

Pode-se observar, também, na Figura 3 baixa concentração de RLO no composto da L3 em relação às outras leiras, no tempo zero de compostagem. Esse comportamento pode ser consequência da baixa concentração de RLO no bagaço de laranja e ao baixo valor de $\mathrm{pH}(4,0)$ da mistura nesse tempo de compostagem pois, segundo Czechowski et al. ${ }^{16}$ a concentração de RLO é altamente sensível ao valor do $\mathrm{pH}$. Esses pesquisadores observaram que a quantificação dos radicais livres em meio alcalino (atmosfera de gás amônia) aumentou de 7 a 15 vezes a concentração dos mesmos, em relação à condição ambiente (atmosfera de ar). Outros trabalhos na literatura ${ }^{13,26}$ também mostram que o $\mathrm{pH}$ tem significativa influência nas medidas de concentração de RLO.

Com o objetivo de avaliar a hipótese da influência da lignina na quantificação dos RLO no composto da L1, fez-se um estudo 
Tabela 1. Valores da relação C/N e CTC/C dos compostos e do índice $\mathrm{A}_{465}$ obtidos dos espectros de fluorescência dos AH extraídos dos compostos da L1, L2 e L3 em função do tempo de compostagem

\begin{tabular}{|c|c|c|c|c|c|c|c|c|c|}
\hline \multirow{2}{*}{$\begin{array}{l}\text { Tempo } \\
\text { (dias) }\end{array}$} & \multicolumn{3}{|c|}{$\mathrm{C} / \mathrm{N}$} & \multicolumn{3}{|c|}{$\mathrm{CTC} / \mathrm{C}\left(\mathrm{mmol}_{\mathrm{c}} \mathrm{g}^{-1}\right)$} & \multicolumn{3}{|c|}{$\mathrm{A}_{465}$} \\
\hline & L1 & L2 & L3 & L1 & L2 & L3 & L1 & L2 & L3 \\
\hline 0 & 112,11 & 36,1 & 50,8 & 0,72 & 0,91 & 1,25 & 1737 & 1842 & 616 \\
\hline 30 & 92,5 & 22,7 & 30,2 & 0,80 & 1,74 & 1,31 & 1776 & 2555 & 1656 \\
\hline 60 & 64,5 & 14,9 & 18,3 & nd & nd & nd & 1665 & 3447 & 3047 \\
\hline 90 & 58,6 & 15,2 & 17,8 & 1,03 & 2,92 & 2,58 & 1638 & 3664 & 3253 \\
\hline 120 & 57,8 & 14,2 & 19,1 & nd & nd & nd & 1576 & 3487 & 3442 \\
\hline 150 & 45,5 & 14,9 & 17,7 & 1,48 & 2,78 & 2,60 & 1547 & 4020 & 3387 \\
\hline 180 & 45,3 & 16,3 & 17,4 & nd & nd & nd & 1697 & 3234 & 3270 \\
\hline 210 & 37,9 & 15,0 & 17,2 & 1,70 & 2,78 & 2,70 & 1555 & 3243 & 3000 \\
\hline
\end{tabular}

nd - não determinado

mais detalhado desse material. Na Figura 4 são apresentadas as concentrações de RLO nos diferentes materiais. Observa-se que a fração lignina apresentou os maiores teores de RLO no tempo 0 e com 210 dias de compostagem. Dessa forma pode-se inferir que o fato da L1 apresentar a maior concentração de RLO, em relação a L2 e L3 (Figura 3), se deve à maior concentração de lignina nesse composto. Também pode-se observar que houve um incremento de, aproximadamente, 2,7 vezes na concentração de RLO na lignina no tempo 210 em relação ao tempo zero de compostagem (Figura 4). Isto sugere que apesar do processo de compostagem ter sido ineficiente na L1 pode ter ocorrido concentração desses radicais devido à perda de estruturas mais lábeis, como as cadeias alifáticas, da matéria orgânica ${ }^{29}$ ou alguma transformação na estrutura da lignina gerando assim uma maior concentração de RLO.

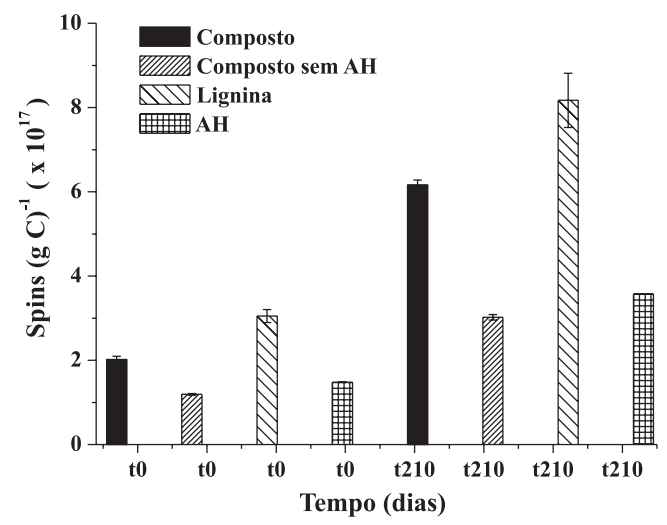

Figura 4. Concentrações de RLO em spins $(\mathrm{g} \mathrm{C})^{-1}\left(\times 10^{17}\right)$ do composto in natura, do composto sem AH (material lavado várias vezes com solução de $\mathrm{NaOH}$ 0,1 mol $\left.\mathrm{L}^{-1}\right)$, da lignina e dos AH da L1 no tempo zero e após os 210 dias de compostagem, determinadas por RPE

Alguns autores consideram que, inicialmente, a lignina é degradada por enzimas extracelulares a unidades menores as quais são susceptíveis à absorção pelas células microbianas onde são parcialmente convertidas a fenóis e quinonas. Essas substâncias são liberadas junto com enzimas oxidativas ao meio ambiente, onde são polimerizadas por mecanismos de formação de radicais livres. ${ }^{30}$

Rimmer $^{31}$ considera que a lignina é um composto polifenólico e é a sua despolimerização uma parte essencial para o processo de degradação e apresenta alto potencial para a formação dos RLO. Esse processo ocorre através da ação de fungos especializados na degradação de lignina e envolve hidrólise enzimática e reações de oxidação por ataque de radicais livres. As quinonas que são produzidas no processo de humificação são provavelmente oriundas dessa despolimerização de polifenóis, como a lignina, que é realizada pelos fungos. ${ }^{16,31}$

Diante dos resultados da concentração dos RLO para o material in natura observa-se que essa análise apresenta valores não confiáveis para o monitoramento do processo de compostagem de material com alta concentração de lignina (como resíduos de poda de árvores na L1) e materiais com grande variação de pH (L3).

\section{Análises de RPE dos AH}

Também foram determinadas a largura de linha do sinal $(\Delta \mathrm{H}) \mathrm{e}$ as concentrações dos RLO nos AH das leiras L1, L2 e L3 a fim de avaliar o uso desses parâmetros na determinação do grau de humificação dos AH extraídos de compostos.

Na Figura 5 são apresentados os espectros dos RLO dos AH da L1 e as larguras de linha dos AH extraídos dos compostos das 3 leiras, em função do tempo de compostagem. Foram observadas variações nas intensidades dos sinais (Figura 5a), mas não se observam grandes variações na largura de linha $(\Delta \mathrm{H})$, em função do tempo (Figura $5 \mathrm{~b}$ ). Esse comportamento corrobora com os dados de Ouatmane et al., ${ }^{32}$ que não observaram alterações significativas nos valores das larguras de linha dos sinais de RPE para AH, extraídos de diferentes compostos. Os autores consideram que esses resultados sugerem baixo grau de aromaticidade, poucas estruturas conjugadas e policondensadas e ocorrência de espécies de radicais livres similares nos AH estudados.

A Figura 6 apresenta as concentrações de RLO nos AH, normalizadas pelo teor de carbono, em função do tempo de compostagem. Observa-se que em todas as leiras houve aumento dos RLO, sendo encontrado os menores valores para a L1. Esse fato pode ser devido à menor formação de $\mathrm{AH}$ nesse composto (L1), por representar um material menos susceptível ao ataque microbiano; enquanto que nas demais leiras a mistura de resíduos, ricos em polissacarídeos e $\mathrm{N}$, favoreceu o processo de humificação, com maior formação de $\mathrm{AH}$.

Para Rimmer ${ }^{31}$ o processo de humificação, pelo qual a matéria orgânica do solo torna-se estabilizada, envolve a decomposição química de resíduos de plantas e animais em produtos como açúcares, compostos amínicos e quinonas, os quais podem ser recombinados por reações de polimerização ou pode haver uma auto-organização dessas moléculas para formar as SH. Esse mesmo mecanismo pode ser considerado no processo de humificação dos compostos.

Jerzykiewicz et al., ${ }^{33}$ estudando a compostagem de resíduo sólido municipal, não observaram alterações significativas na concentração de RLO no material in natura. Porém, observaram aumento da concentração desses radicais livres nas frações AH e AF de alguns compostos. Eles observaram que para os $\mathrm{AH}$ a concentração máxima de RLO foi em, aproximadamente, 40 dias de compostagem seguida 

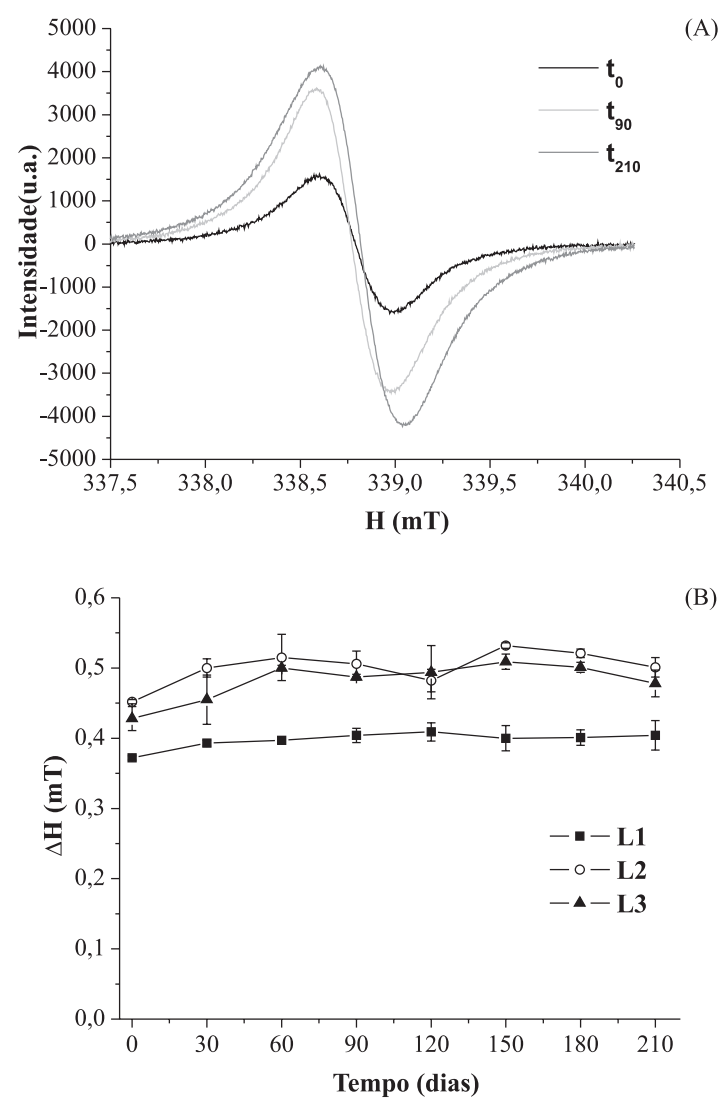

(B)

Figura 5. Sinais de RLO dos AH extraídos da L1 em função do tempo de compostagem (a), largura de linha tomada pico a pico $(\Delta H)$ do sinal de RLO dos AH extraídos dos compostos da L1, L2 e L3 em função do tempo de compostagem $(b)$

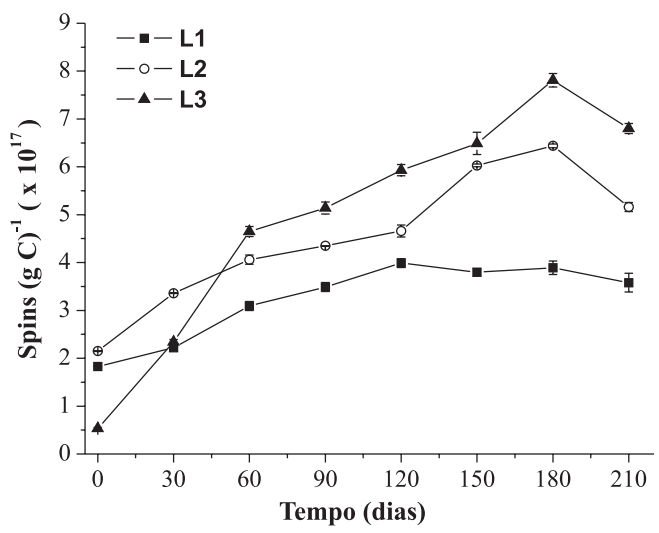

Figura 6. Concentrações dos RLO em spins $(\mathrm{g} \mathrm{C})^{-1}\left(\times 10^{17}\right)$ dos AH extraídos das leiras L1, L2 e L3 em função do tempo de compostagem, determinadas por $R P E$

de queda nos valores desses radicais. Nesse trabalho foram observadas concentrações máximas de RLO em 180 dias, seguida de queda das concentrações desses radicais (Figura 6).

Apesar das concentrações de RLO dos AH extraídos da L1 terem apresentado incremento em função do tempo de compostagem, elas mostraram os menores valores, a partir de 30 dias, em comparação com as leiras L2 e L3 (Figura 6), sendo esse resultado mais coerente com os apresentados na Tabela 1, onde foi observado que a L1 não apresentou características de material humificado.

Nesse trabalho confirmou-se que para haver a formação dos AH os resíduos devem apresentar boas condições para a efetiva ação dos micro-organismos, sendo um dos pré-requisitos a relação $\mathrm{C} / \mathrm{N}$ inicial próxima de 35. Pôde-se observar que na L1 houve alguma transformação da lignina (pelos resultados de RPE do composto, mostrado na Figura 4), porém, pelos demais resultados obtidos (Tabela 1) não se pode inferir sobre a humificação desse composto.

\section{CONCLUSÕES}

Houve aumento na concentração dos radicais livres orgânicos do tipo semiquinona (RLO) durante o processo de compostagem, em todas as leiras analisadas, porém no material in natura da L1 observou-se um aumento mais significativo que nas demais leiras, sugerindo que esse composto seria o mais humificado. Esse fato foi contraditório em relação ao processo de humificação dessa leira, tendo em vista que a relação $\mathrm{C} / \mathrm{N}, \mathrm{CTC} / \mathrm{C}$, dados de fluorescência e temperatura não mostraram evolução do processo na L1. Um estudo mais detalhado desse composto permitiu constatar que a lignina é a principal fonte dos RLO. Assim, a determinação desses radicais em material in natura, que contenha alto teor de lignina, como poda de árvores, ou grande variação de $\mathrm{pH}$, como no caso da leira com bagaço de laranja (L3), não é um bom indicador para monitorar o processo de humificação na compostagem. Para a análise por RPE deve-se fazer a extração da fração AH dos compostos a serem analisados.

\section{AGRADECIMENTOS}

À FAPESP (Projeto: 03/06097 - 4) e ao CNPq pelo suporte financeiro e bolsa concedida.

\section{REFERÊNCIAS}

1. Provenzano, M. R.; Oliveira, S. C.; Silva, M. R. S.; Senesi, N.; J. Agric. Food Chem. 2001, 49, 5874.

2. Bernal, M. P.; Paredes, C.; Sánchez-Monedero, M. A.; Cegarra, J.; Bioresour. Technol. 1998, 63, 91

3. Browning, B. L.; Methods of wood chemistry, John Wiley: New York, 1967.

4. Fengel, D.; Wegener, G.; Wood chemistry, ultrastructure, reactions, Walter de Gruyter: Berlin, 1984.

5. López, M. J.; Elorrieta, M. A.; Vargas-Garcia, M. C.; Suarez-Estrella, F.; Moreno, J.; Bioresour. Technol. 2002, 81, 123.

6. Rex, R. W.; Nature 1960, 188, 1185.

7. Steelink, C.; Tollin, G.; Biochim. Biophys. Acta 1962, 59, 25.

8. Saab, S. C.; Martin-Neto, L.; J. Braz. Chem. Soc. 2008, 19, 413.

9. Saab, S. C.; Martin-Neto, L.; J. Braz. Chem. Soc. 2004, 15, 34.

10. Bayer, C.; Martin-Neto, L.; Mielniczuk, J.; Saab, S. C.; Milori, D. M. B. P.; Bagnato, V. S.; Geoderma 2002, 105, 81.

11. Olk, D. C.; Brunetti, G.; Senesi, N.; Soil Sci. Soc. Am. J. 2000, 64, 1337.

12. Martin-Neto, L.; Rossel, R.; Sposito, G.; Geoderma 1998, 81, 305.

13. Senesi, N.; Application of electron spin resonance (ESR) spectroscopy in soil chemistry, Springer-Verlag: New York, 1990.

14. Senesi, N. Em Humus, its structure and role in agriculture and environment; Kubat, J., ed.; Elsevier Science Publishers: Amsterdan, 1992.

15. Novotny, E. H.; Martin-Neto, L.; Geoderma 2002, 106, 305.

16. Czechowski, F.; Golonka, I.; Jezierski, A.; Spectrochim. Acta, Part A 2004, 60, 1387

17. Malavolta, E.; Vitti, G. C.; Oliveira, S. A.; Avaliação do estado nutricional das plantas: princípios e aplicações, Associação Brasileira para Pesquisa da Potassa e do Fosfato: Piracicaba, 1989.

18. Rodella, A. A.; Alcarde, J. C.; Sci. Agric. 1994, 51, 556.

19. Williams, S.; Official methods of analysis of the associations official analytical chemists, $14^{\text {th }}$ ed., AOAC: Arlington, 1984. 
20. Swift, R. S. Em Methods of Soil Analysis Part 3; Sparks, D. L., ed., Soil Sci. Soc. Am.: Madison, 1996.

21. McPhie, P. Em Methods in enzymology; Jacoby, W. B., ed.; Academic Press: New York, 1971.

22. Technical Association of the Pulp and Paper Industry; TAPPI test methods, TAPPI Press: Atlanta, 1998.

23. Martin-Neto, L.; Traghetta, D. G.; Vaz, C. M. P.; Crestana, S.; Sposito, G.; J. Environ. Qual. 2001, 30, 520.

24. Poole, C.; Farach, H. T.; Theory of magnetic resonance, WileyInterscience: New York, 1972.

25. Milori, D. M. B. P.; Martin-Neto, L.; Bayer, C.; Mielniczuk, J.; Bagnato, V. S.; Soil Sci. 2002, 167, 739 .
26. Senesi, N.; Schnitzer, M.; Soil Sci. 1977, 123, 224.

27. Tomati, U. ; Belardinelli, M. ; Andreu, M. ; Galli, E. ; Capitani, D. ; Proietti, N. ; Simone, C.; Waste Manage. Res. 2002, 20, 389.

28. http://extranet.agricultura.gov.br/sislegis-consulta/consultarLegislacao. do, acessada em Agosto 2009.

29. Saab, S. C.; Martin-Neto, L.; Quim. Nova 2003, 26, 497.

30. Tuomela, M.; Vikman, M.; Hatakka, A.; Itavaara, M.; Bioresour. Technol. 2000, 72, 169.

31. Rimmer, D. L.; Eur. J. Soil Sci. 2006, 57, 91.

32. Ouatmane, A.; D'orazio, V.; Hafidi, M.; Senesi, N.; Compost Sci. Land Util. 2002, 10, 39 .

33. Jerzykiewicz, M.; Drozd, J.; Jezierski, A.; Chemosphere 1999, 39, 253. 